7 Garcha DS, Thurston SJ, Patel AR, et al. Changes in prevalence and load of airway bacteria using quantitative PCR in stable and exacerbated COPD. Thorax 2012; 67: 1075-1080.

8 Han MK, Huang YJ, Lipuma JJ, et al. Significance of the microbiome in obstructive lung disease. Thorax 2012; 67: 456-463.

9 Erb-Downward JR, Huffnagle GB, Martinez FJ. The microbiota in respiratory disease. Am J Respir Crit Care Med 2012; 185: 1037-1038.

10 Human Microbiome Project Consortium. Structure, function and diversity of the healthy human microbiome. Nature 2012; 486: 207-214.

Boersma WG. Antibiotics in acute exacerbations of COPD: the good, the bad and the ugly. Eur Respir J2012; 40: 1-3. Sialer S, Adamantia L, Guerrero M, et al. Relation between chronic obstructive pulmonary disease and antibiotics. Curr Infect Dis Rep 2012; 14: 300-307.

\title{
Infantile growth velocity and later asthma/wheeze: GENESIS and the Healthy Growth Study
}

\author{
To the Editor:
}

Accumulating evidence suggests that wheezing illnesses have at least part of their origin in the infantile period, wherein septal restructuring and alveolar formation ensue. Therefore, throughout infancy, genetic and environmental factors could affect bronchial development and future airway function. On this basis, we hypothesised that differential growth velocity, within the first 6 months of life, could be diversely linked to paediatric wheezing illnesses. Establishing such an interaction still eludes us, due to limited and conflicting evidence [1-6] and age-specific differences in bronchial function/structure [3]. We, therefore, opted to investigate a potential correlation in two populations of children of different ages, the Growth, Exercise and Nutrition Epidemiological Study In preSchoolers (GENESIS) study and the Healthy Growth Study cohorts (preschool and late childhood, respectively); thereby, we also appraised any age-definable discrepancies. These cross-sectional studies involved children aged 2-5 years attending nurseries in five Greek counties (GENESIS study) [7] and children aged 9-13 years, attending primary schools in four Greek counties (Healthy Growth Study) [8]. Sampling of nurseries/schools was stratified by parents' educational level and total student population in the respective municipality and their selection was random, as previously described $[7,8]$. By using the parents' educational level as a proxy for socioeconomic level (SEL), we opted to compile a sample that was largely representative of the SEL level of Greek children. Specifically, municipalities were classified in three categories of different SELs i.e. Higher, Medium and Lower (Census 2001 [9]). Subsequently, certain municipalities were randomly selected from each one of these three SEL groups; their number was proportional to the size of each group's preschool/pre-adolescent population. Finally, schools were randomly selected from each municipality; their number was proportional to the municipality's schoolchildren population. Parental consent was acquired and the studies were approved by the Greek Ministry of Education and the Ethics Committee of the Harokopio University, Athens, Greece. Socio-demographic and perinatal data was collected and body fat assessment/anthropometric measurements were conducted during school interviews. The parents provided the researchers with their children's health books wherein (as part of standard practice in Greece) data from routine paediatrician-conducted measurements of infantile weight and length were recorded; this information was used to estimate weightfor-length z-scores based on the World Health Organization (WHO) growth charts. The z-score difference between birth and 6 months of age was used to classify children into the following categories: retarded growth velocity ( $\leqslant 1 \mathrm{z}$-score difference), normal growth velocity $(-1 \leqslant 1 \mathrm{z}$-score difference), and rapid growth velocity ( $>1 \mathrm{z}$-score difference). Potential atopic predisposition of the GENESIS participants was evaluated through the question: "Did a doctor ever tell you that your child has atopic dermatitis?". Asthma outcomes were defined from the International Study of Asthma and Allergies in Childhood (ISAAC) core questionnaire. 1) Current wheeze (did your child have wheezing or whistling in the chest in the past 12 months?). 2) Ever wheeze (did your child have wheezing or whistling in the chest at any point in time?). 3) Asthma ever (did your child ever have asthma diagnosed by a doctor?). Complete data were available from 1668 children in the GENESIS study and 1867 children in the Healthy Growth Study. Univariate logistic regression analyses were conducted and followed by multivariate logistic regression analyses, whereby we adjusted for a wide array of potential confounding/intervening variables (table 1). The 


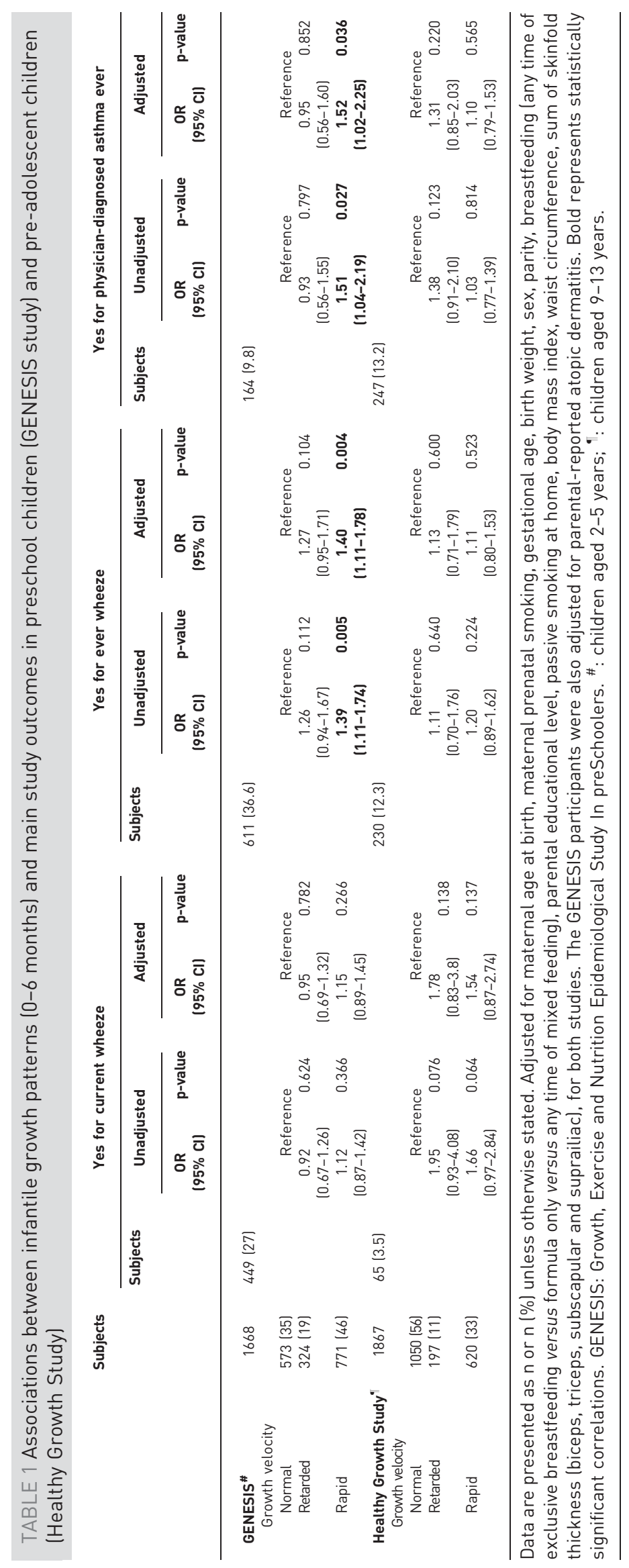


statistical package for social sciences (SPSS; IBM Corporation, Armonk, NY, USA), version 20.0 was used and a two-tailed $\mathrm{p}<0.05$ was considered significant.

The correlations uncovered by the univariate analyses were not meaningfully altered following the incorporation of the confounders in the models. Table 1 presents the unadjusted and adjusted associations of the infantile growth patterns with the main outcomes. In preschool children, rapid growth velocity in the first 6 months of life was positively associated with ever wheeze $(\mathrm{p}=0.005)$ and asthma ever $(\mathrm{p}=0.036)$ but not with current wheeze, following adjustment. In preadolescents, neither rapid nor retarded growth velocity was associated with any outcome $(\mathrm{p}>0.05)$. Current wheeze did not correlate to any infantile growth pattern, in either population.

Investigations into infantile growth patterns were kindled by the "Barker" hypothesis, which maintains that retarded growth in utero could cause rapid infantile catch-up (compensatory) growth and have detrimental repercussions on later health. Notwithstanding a potential deleterious effect of intrauterine growth restrain per se on later asthma/wheeze, catch-up postnatal growth may instead be the main culprit. Although such mechanisms are not yet clear, they conceivably rely on a potential "programming", whereby various infantile exposures including early-life growth could exert long-term effects on tissue function and dictate emergence of relevant pathology.

Pertinent evidence regarding asthma is limited, as only recently a potential infantile growth velocity/asthma relation has attracted attention. Investigators have reported a positive correlation of rapid infantile weight gain with wheeze at the age of 1,3 and 4 years $[1,3,10]$, with asthma up to the age of 10 years $[4,11]$, with preschool asthma exacerbations [2], and with adult wheeze [5]. In a recent report in the European Respiratory Journal, VAN DER GUGTEN et al. [6] showed that rapid infantile growth velocity is a risk factor for asthma/decreased lung function, up to the age of 5 years. This evidence is in keeping with our own findings of a positive association of rapid infantile growth velocity with ever wheeze and asthma incidence in preschool children. However, it is at odds with our findings in preadolescents, as in this age range we failed to show any such correlation. Age-specific variability in wheeze causation could partly reconcile these discrepancies. For instance, post-viral wheeze is prevalent in preschool children, whereas atopic wheeze is predominant in older children; hence, since rapid growth velocity was associated with asthma/wheeze only in the preschool age, it stands to reason that it may be linked to post-viral wheeze. Indeed, adjustment for atopic dermatitis in preschool children did not alter these correlations, suggesting that atopy does not perturb in this mechanism. In fact, infantile rapid growth velocity may act as a proxy for a predisposition to illness (and hence to post-illness wheeze), as it could compensate for the deceleration caused by illnesses during infancy [4]. This could also explain the lack of an association of rapid growth velocity with preschool current wheeze: ever wheeze (which was linked with rapid growth velocity in this cohort), could be predominant in infancy (due to infantile illness), rather than be current. Such mechanisms merit further research through prospective studies.

Our study's strengths include its population-based design, findings from two discrete cohorts of children of different ages and control for several confounders. We adjusted for short gestational age and low birth weight, both of which are associated with asthma [10]. Low birth weight could also act as a surrogate for retarded fetal growth; hence, making allowance for it, minimises the possibility that the associations we reported were mediated by prenatal growth restraint $[1,10]$. We also adjusted for current fat mass indices: body mass index, waist circumference and sum of skinfold thickness; insofar as accelerated infantile growth could be a risk factor for later obesity, controlling for them refutes the possibility that our findings are underpinned by an asthma-facilitating effect of excess fat mass. We further controlled for maternal prenatal smoking, a risk factor for fetal growth retardation [10] and preschool wheeze, in addition to maternal age at birth, passive smoking at home, parity, sex, breastfeeding and parental educational level, all of which modify asthma risk. Nevertheless, the retrospective design imposes certain limitations, such as potential recall and misclassification bias. Furthermore, atopy was not adjusted for through objective means, but rather through doctor-diagnosed atopic dermatitis, which is only one of many possible marks of an atopic phenotype. However, in light of the well-established temporal course of the atopic march, we feel that skin manifestations are a relevant marker of atopy for a preschool-aged cohort. In any case, an association of infantile growth patterns with atopy per se, has been contested in several studies $[1,3,5,10]$.

In conclusion, we report that rapid growth velocity in the first 6 months of life is linked to increased wheeze and asthma incidence in preschool children but not preadolescents. Importantly, this correlation is independent of current fat mass and birth weight, suggesting that it is unrelated to obesity and in utero growth retardation, respectively. Confinement of this association in the preschool age and lack of interference of atopy, imply a link of rapid growth velocity with post-viral wheeze. We believe that further research into these mechanisms could have important implications in preschool-wheeze prevention. 
George V. Guibas ${ }^{1}$, Yannis Manios ${ }^{2}$, George Moschonis ${ }^{2}$, Paraskevi Xepapadaki ${ }^{1}$, Eirini Roumpedaki ${ }^{1}$,

Christina-Paulina Lambrinou ${ }^{2}$ and Nikolaos G. Papadopoulos ${ }^{1}$

${ }^{1}$ Allergy Dept, 2nd Pediatric Clinic, University of Athens, Athens, and ${ }^{2}$ Dept of Nutrition and Dietetics, Harokopio

University of Athens, Athens, Greece.

Correspondence: N. G. Papadopoulos, 41 Fidippidou Street, Athens, 115 27, Greece. E-mail: ngp@allergy.gr

Received: July 262013 | Accepted after revision: Dec 192013 | First published online: Feb 202014

Conflict of interest: Disclosures can be found alongside the online version of this article at www.erj.ersjournals.com

\section{References}

1 Pike KC, Crozier SR, Lucas JS, et al. Patterns of fetal and infant growth are related to atopy and wheezing disorders at age 3 years. Thorax 2010; 65: 1099-1106.

2 Paul IM, Camera L, Zeiger RS, et al. Relationship between infant weight gain and later asthma. Pediatr Allergy Immunol 2010; 21: 82-89.

3 Taveras EM, Rifas-Shiman SL, Camargo CA Jr, et al. Higher adiposity in infancy associated with recurrent wheeze in a prospective cohort of children. J Allergy Clin Immunol 2008; 121: 1161-1166.

4 Flexeder C, Thiering E, Brüske I, et al. Growth velocity during infancy and onset of asthma in school-aged children. Allergy 2012; 67: 257-264.

5 Rona RJ, Smeeton NC, Bustos P, et al. The early origins hypothesis with an emphasis on growth rate in the first year of life and asthma: a prospective study in Chile. Thorax 2005; 60: 549-554.

6 Van der Gugten AC, Koopman M, Evelein AM, et al. Rapid early weight gain is associated with wheeze and reduced lung function in childhood. Eur Respir J 2012; 39: 403-410.

7 Manios Y. Design and descriptive results of the "Growth, Exercise and Nutrition Epidemiological Study In preSchoolers": the GENESIS study. BMC Public Health 2006; 6: 32.

8 Moschonis G, Tanagra S, Vandorou A, et al. Social, economic and demographic correlates of overweight and obesity in primary-school children: preliminary data from the Healthy Growth Study. Public Health Nutr 2010; 13: $1693-1700$.

9 National Statistical Service of Greece Population and Housing Census 2001. www.unece.org Date last updated: February 2003. Date last accessed: February 2014.

10 Sonnenschein-van der Voort AM, Jaddoe VW, Raat H, et al. Fetal and infant growth and asthma symptoms in preschool children: the Generation R Study. Am J Respir Crit Care Med 2012; 185: 731-737.

11 Rzehak P, Wijga AH, Keil T, et al. Body mass index trajectory classes and incident asthma in childhood: results from 8 European Birth Cohorts - a Global Allergy and Asthma European Network initiative. J Allergy Clin Immunol 2013; 131: 1528-1536.

\section{Replacing smear microscopy for the diagnosis of tuberculosis: what is the market potential?}

\section{To the Editor:}

Sputum smear microscopy (SSM) has been the cornerstone of tuberculosis (TB) diagnosis, and is mainly performed in peripheral microscopy centres attached to primary health centres where TB therapy can be administered. Although SSM is inexpensive and easy to perform with a limited infrastructure, the shortcomings are its relatively low sensitivity and its inability to detect drug-resistance. Thus, there is a need for a more sensitive technology that can replace microscopy $[1,2]$.

Several next-generation molecular diagnostics are under development with the specific intention of use in microscopy centres [3-5]. In a recent survey of 22 high-burden countries (HBCs), we showed that the conditions, equipment and expertise present in microscopy centres are challenging and need to be considered by product developers [6]. While the Xpert MTB/RIF (Cepheid Inc., Sunnyvale, CA, USA) assay is accurate, endorsed by the World Health Organization and is being implemented in many countries, it was not designed for use in peripheral microscopy centres $[7,8]$. To assist product developers working on tests for use in microscopy centres, we have outlined the desirable test characteristics [1]. 